\title{
On Some Reliability Properties of Mean Inactivity Time under Weighing
}

\author{
Neeraj Gandotra \\ Jaypee University of \\ Information Technology, \\ Waknaghat, H.P., INDIA \\ $\mathrm{PIN}-173234$
}

\author{
Rakesh Kumar Bajaj \\ Jaypee University of \\ Information Technology, \\ Waknaghat, H.P., INDIA \\ $\mathrm{PIN}-173234$
}

\author{
Nitin Gupta \\ Jaypee University of \\ Information Technology, \\ Waknaghat, H.P., INDIA \\ $\mathrm{PIN}-173234$
}

\begin{abstract}
In the present communication, we discuss some reliability properties of mean inactivity time order and study the preservation of mean inactivity time under some weight function. Weighted Distribution methods arise in the context of research related to reliability, data gathering, ecology, inference, modeling, bio-medicine and several other areas. In general, weighted distributions have wide role for the analysis of life time data.
\end{abstract}

\section{General Terms}

Reliability, Stochastic Orders, Stochastic aging

\section{Keywords}

Mean Inactivity Time, shifted likelihood ratio order, residual life

\section{INTRODUCTION}

By the method of ascertainment, the concept of weighted distribution has been introduced by C.R. Rao in 1963 (see Rao, [1] ). Weighted distribution have been widely used as a tool in various practical problems in the selection of appropriate models for observed data drown without a proper frame, analysis of data relating to human populations and wild life management, investigation of human heredity, line transcend sampling and renewal theory, study of statistical ecology, albinism and reliability modeling. The properties of weighted random sample corresponding to original random sample is required to study when the observations can be recorded as a weighted random sample with some weight attached to the original random sample.

Let $f(\cdot)$ be the probability density function of original random variable $X$. Let $w_{1}(\cdot): R \rightarrow[0, \infty)$, where $R=(-\infty, \infty)$ and the recovered random variable be $X_{w_{1}}$ with the probability density function given by

$$
f_{w_{1}}(x)=\frac{w_{1}(x) f(x)}{w_{1}} ; x \in R
$$

where $\quad w_{1}=E\left(w_{1}(X)\right)>0$.

The random variable $X_{w_{1}}$ is called the weighted version of $X$ and its distribution in relation to that of $X$ is called the weighted distribution of $X$ with weighted function $w_{1}(\cdot)$. Jain et. al. [2], Nand \& Jain [3], Misra et. al. [4], Barlow et. al. [5], and Bartoszewicz et.al. [6] have studied the reliability properties of weighted distributions in relation to corresponding reliability measures of parent distributions. Here, we further derive some results on preservation of mean inactivity time order and mean inactivity time ordering by weighted distributions.

Let $w_{1}, w_{2}$ be two functions where $w_{i}: R \rightarrow R^{+}, i=1,2$ such that $0<E\left[w_{1}(X)\right]<\infty, \quad 0<E\left[w_{2}(Y)\right]<\infty \quad$ and $w_{1}=E\left[w_{1}(X)\right], w_{2}=E\left[w_{2}(Y)\right]$. Let $X_{w_{1}}$ and $Y_{w_{2}}$ be the weighted versions of $X$ and $Y$ with weight functions $w_{1}(\cdot)$ and $w_{2}(\cdot)$ respectively. Then $X_{w_{1}}$ and $Y_{w_{2}}$ have probability density functions given by

$$
f_{w_{1}}(x)=\frac{w_{1}(x) f(x)}{w_{1}}, \quad x \in R
$$

and

$$
g_{w_{2}}(x)=\frac{w_{2}(x) g(x)}{w_{2}}, \quad x \in R
$$

respectively.

Let $F_{1}(x)\left(G_{2}(x)\right), x \in R$ be the distribution function of $X_{w_{1}}\left(Y_{w_{2}}\right)$ and let

$$
\bar{F}_{1}(x)=1-F_{1}(x)\left(\bar{G}_{2}(x)=1-G_{2}(x)\right), \quad x \in R
$$

be the survival function of $X_{w_{1}}\left(Y_{w_{2}}\right)$. 
Let us consider a random variable $X$ with absolutely continuous distribution function

$$
F(x)=P(X \leq x), x \in R,
$$

survival (reliability) function

$$
\bar{F}(x)=1-F(x), x \in R
$$

and the probability density function $f(x), x \in R$, to provide definitions of notions of reliability classes, statistical dependence, stochastic orders etc.

Let the reverse failure rate ( $\mathrm{rfr}$ ) of a random variable $X$ is given by

$$
r_{X}(x)=\frac{f(x)}{F(x)}, x>0
$$

The residual life of random variable $X$ with fixed age/time $t$ $(t>0)$ is

$$
X_{t}=(X-t \mid X>t)
$$

and inactivity time of random variable $X$ with fixed age/time $s$ $(s>0)$ is

$$
X_{(s)}=(s-X \mid X \leq s)
$$

The conditional distribution of $X-t$ given $X>t$ and $s-X$ given $X \leq s$ are the distributions of $X_{t}$ and $X_{(s)}$ respectively. The mean residual life function and mean inactivity time function when the random variable $X$ has finite mean are defined as

$$
m_{X}(t)=E\left(X_{t}\right)=\frac{\int_{t}^{\infty} \bar{F}(u) d u}{\bar{F}(t)}
$$

and

$$
\mu_{X}(s)=E\left(X_{(s)}\right)=\frac{\int_{-\infty}^{s} F(u) d u}{F(s)}
$$

respectively.

Next, we introduce following definitions which are standard in literature (Refer Shaked, M. and Shanthikumar [7] and Ahmad and Kayid [8] ) :

\section{Definitions:}

(a) Let $\Omega=(a, b) \subseteq R$ where $-\infty \leq a<b \leq \infty$ and $h: \Omega \rightarrow R^{+}$. Then the function $h(\cdot)$ is said to be log-concave (log-convex) on $\Omega$ if, $\forall x, y \in \Omega$ and $\forall \alpha \in(0,1)$,

$h(\alpha x+(1-\alpha) y) \geq(\leq)(h(x))^{\alpha}(h(y))^{1-\alpha}$.

(b) Random variable $X$ is said to have decreasing (increasing) reversed failure rate (DRFR (IRFR)) if $F(\cdot)$ is log-concave (log-convex) on $0, \infty$, or equivalently if the reversed failure rate function $r_{X}(\cdot)$ is decreasing (increasing) on $0, \infty$.

(c) Random variable $X$ is said to have decreasing (increasing) mean residual life (DMRL (IMRL)) if $\int_{x}^{\infty} \bar{F}(t) d t$ is log-concave (log-convex) on $0, \infty$.

(d) Random variable $X$ is said to have increasing (decreasing) mean inactivity time (IMIT (DMIT)) if $\int_{0}^{x} F(u) d u$ is log-concave (log-convex) on $0, \infty$ or equivalently if the mean inactivity time function $\mu_{X}(\cdot)$ is decreasing (increasing) on $0, \infty$.

(e) Random variable $X$ is said to be smaller then $Y$ in the down shifted likelihood ratio order (written as $\left.X \leq_{l r \downarrow} Y\right)$ if $\frac{g(t+x)}{f(t)}$ is increasing in $t \geq 0$ for all $x \geq 0$, where $f$ and $g$ denote the density functions of $X$ and $Y$ respectively.

(f) Random variable $X$ is said to be smaller than random variable $Y$ in the reversed failure rate ordering (written as $\left.X \leq_{r f r} Y\right)$ if

$$
G(s) F(t) \leq G(t) F(s),
$$

whenever

$$
-\infty<s<t<\infty
$$

or equivalently, if

$$
r_{X}(x) \leq r_{Y}(x), \forall x \in(0, \infty)
$$

(g) Random variable $X$ is said to be smaller then random variable $Y$ in the mean inactivity time ordering $\left(X \leq_{m i t} Y\right)$ if 


$$
\begin{aligned}
{\left[\int_{-\infty}^{s} G(u) d u\right]\left[\int_{-\infty}^{t} F(u) d u\right] \leq } \\
{\left[\int_{-\infty}^{s} F(u) d u\right]\left[\int_{-\infty}^{t} G(u) d u\right] }
\end{aligned}
$$

whenever

$$
-\infty<s<t<\infty,
$$

or equivalently,

$$
\mu_{X}(t) \geq \mu_{Y}(t), \forall t \in(0, \infty) .
$$

\section{AGING PROPERTIES STOCHASTIC DEPENDENCE}

AND

Let $X$ and $Y$ be non-negative random variables.

Lemma 2.1 (Misra et. al. [4], Theorem 2.3(a)).

If $X$ has DRFR, $w_{1}(\cdot)$ is decreasing on $0, \infty$ and log-concave on $0, \infty$, then $X_{w_{1}}$ has DRFR.

Lemma 2.2 (Misra et. al. [4], Theorem 3.2(c)).

If $X \leq_{r f r} Y, \quad w_{1}(t)$ is decreasing on $0, \infty$ and $w_{2}(t) / w_{1}(t)$ is increasing on $0, \infty$, then $X_{w_{1}} \leq_{r f r} Y_{w_{2}}$.

Here we present the condition on weight function $w$. under which such preservation of property of IMIT under weighing is possible.

Theorem 2.1:

$X_{w_{1}}$ has IMIT if $X$ has IMIT and $A_{1}(\cdot)$ is decreasing and log-convex on $0, \infty$ where $A_{1}(x)=E \quad w_{1}(x) \mid X \leq x$.

Proof:

Let $Z_{1}$ and $Z_{2}$ be random variables having probability density function

$$
f_{Z_{1}}(x)=\frac{F(x)}{\int_{0}^{\infty} F(u) d u},
$$

and

$$
f_{Z_{2}}(x)=\frac{F_{w_{1}}(x)}{\int_{0}^{\infty} F_{w_{1}}(u) d u},
$$

respectively.

$Z_{1}$ has weighted version $Z_{2}$ with weight function $A_{1}(\cdot)$. The random variable $Z_{1}$ has DRFR since $X$ has IMIT. Under the premise of the contention, using Lemma 2.1, it follows that random variable $Z_{2}$ has DRFR which follows that $X_{w_{1}}$ has IMIT.

Consider

$$
F_{1}(x)=\frac{A_{1}(x) F(x)}{w_{1}} ;
$$

where

$$
A_{1}(x)=E\left[w_{1}(X) \mid X \leq x\right] .
$$

From above, it is clear that $X_{w_{1}}$ has DRFR (IRFR), if $X$ has DRFR (IRFR).

The following theorem provides conditions on the weight function $w_{1}(\cdot)$ and the mean inactivity time function $\mu_{X}(t)$, under which a random variable $X$ having IMIT, yield a weighted version which is DRFR (and hence IMIT)

Theorem 2.2:

$X_{w_{1}}$ has DRFR if $X$ has IMIT, $w_{1}(\cdot)$ is decreasing and log-concave on $0, \infty$ and the mean inactivity time function $\mu_{X}(t)$ is log-convex on $0, \infty$.

Proof:

In view of Lemma 2.1, it is enough to show that $X \leq_{r f r} Z_{1}$ where $Z_{1}$ has probability density function $f(t-\theta)$, i.e., $X$ has DRFR and mean inactivity time function $\mu_{Z_{1}}(t)=\mu_{X}(t-\theta)$. 
Consider

$$
\begin{aligned}
& r_{Z_{1}}(t)-r_{X}(t)= \frac{1-\mu_{Z_{1}}^{\prime}(t)}{\mu_{Z_{1}}(t)}-\frac{1-\mu_{X}^{\prime}(t)}{\mu_{X}(t)} \\
&= {\left[\frac{\mu_{X}^{\prime}(t)}{\mu_{X}(t)}-\frac{\mu_{X}^{\prime}(t-\theta)}{\mu_{X}(t-\theta)}\right] } \\
&+\left[\frac{1}{\mu_{X}(t-\theta)}-\frac{1}{\mu_{X}(t)}\right] \\
& \geq 0,
\end{aligned}
$$

since $\mu_{X}(t)$ is log-convex on $0, \infty$ and $X$ has IMIT.

Therefore, $X \leq_{r f r} Z_{1}$ and hence $X$ has DRFR. Now contention follows using Lemma 2.1.

\section{Theorem 2.3:}

$X_{w_{1}} \leq_{m i t} Y_{w_{2}} \quad$ if $\quad X \leq_{m i t} Y, \quad A_{1}(\cdot) \quad$ is decreasing and $A_{2}(\cdot) / A_{1}(\cdot)$ is increasing on $0, \infty$, where

$$
A_{1}(x)=E \quad w_{1}(X) \mid X \leq x
$$

and $\quad A_{2}(x)=E \quad w_{2}(Y) \mid Y \leq x$.

\section{Proof:}

We have

$$
F_{w_{1}}(x)=\frac{A_{1}(x) F(x)}{w_{1}}
$$

and

$$
G_{w_{2}}(x)=\frac{A_{2}(x) G(x)}{w_{2}} .
$$

Let $X^{*}$ and $Y^{*}$ be random variables with probability density functions given by

$$
f_{X^{*}}(x)=\frac{F(x)}{\int_{0}^{\infty} F(u) d u}
$$

and

$$
f_{Y^{*}}(x)=\frac{G(x)}{\int_{0}^{\infty} G(u) d u}
$$

respectively.

Now $X \leq_{m i t} Y$ implies that $X^{*} \leq_{r f r} Y^{*}$. Let $X_{A_{1}}^{*}$ and $Y_{A_{2}}^{*}$ be weighted version of $X^{*}$ and $Y^{*}$ with weight functions $A_{1}(\cdot)$ and $A_{2}(\cdot)$ respectively. Hence by Lemma 2.2 ,

$$
X^{*} \leq_{r f r} Y^{*}
$$

Also, $X^{*} \leq_{r f r} Y^{*}$, if and only if $X_{w_{1}} \leq_{m i t} Y_{w_{2}}$.

\section{Proposition:}

$A_{1}(\cdot)$ is increasing (decreasing) if $w_{1}(\cdot)$ is increasing (decreasing).

\section{Proof:}

Consider

$$
F(x) w(x)-\int_{0}^{x} w(t) f(t) d t \geq(\leq) 0
$$

if and only if $\quad A_{1}{ }^{\prime}(x) \geq(\leq) 0$.

It may be noted that $w_{1}(\cdot)$ is increasing (decreasing) implies that $A_{1}(\cdot)$ is increasing (decreasing). Hence, the result follows by using the above argument.

Corollary: If $X \leq_{\text {mit }} Y, w_{1}(\cdot)$ is decreasing and $w_{2}(\cdot)$ is increasing on $0, \infty$, then $X_{w_{1}} \leq_{m i t} Y_{w_{2}}$.

Example: If $f(x)=e^{-x}$ and $w_{1}=x^{\alpha_{1}-1}$ where $\alpha_{1}>1$, $w_{2}=x^{\alpha_{2}-1}$ where $\alpha_{2}<1$ then $X_{w_{1}} \leq_{m i t} Y_{w_{2}}$.

\section{Theorem 2.4:}

If $f(\cdot)$ is log-convex and $w(\cdot)$ is increasing on $[0, \infty)$, then $X \leq_{l r \downarrow} X_{w}$. 


\section{Proof:}

For fixed $a>0$, consider

$$
\begin{gathered}
\frac{f_{w}(x+a)}{f(x)}=\frac{w(x+a) f(x+a)}{E[w(X+a)] f(x)} \\
=\frac{1}{E[w(X+a)]} \cdot w(x+a) \cdot \frac{f(x+a)}{f(x)},
\end{gathered}
$$

which is increasing function, since $f(\cdot)$ is log-convex and $w(\cdot)$ is increasing. Hence $X \leq_{l r \downarrow} X_{w}$.

\section{Theorem 2.5:}

If $X \leq_{l r \downarrow} Y$ and $w(\cdot)$ is log-convex, then $X_{w} \leq_{l r \downarrow} Y_{w}$.

\section{Proof:}

For fixed $a>0$, consider

$$
\begin{gathered}
\frac{g_{w}(x+a)}{f_{w}(x)}=\frac{w(x+a) g(x+a)}{E[w(Y+a)]} \cdot \frac{E[w(x)]}{w(x) f(x)} \\
=\frac{E[w(x)]}{E[w(Y+a)]} \cdot \frac{w(x+a)}{w(x)} \cdot \frac{g(x+a)}{f(x)},
\end{gathered}
$$

which is an increasing function, since $w(\cdot)$ is log-convex and

$$
X \leq_{l r \downarrow} Y \text {. Hence } X_{w} \leq_{l r \downarrow} Y_{w} \text {. }
$$

\section{CONCLUSION}

In the context of reliability and life testing problems, we obtain reliability properties of mean inactivity time under weighting.
The conditions under which such results have been obtained are described in this paper. Further, the conditions of stochastic comparison of weighted distributions in terms of mean inactivity time and shifted likelihood ratio order has been obtained.

\section{ACKNOWLEDGMENTS}

The authors are thankful to the anonymous referees for their valuable comments and suggestions. The authors sincerely appreciate the efforts of the Editor in Chief of the journal.

\section{REFERENCES}

[1] Rao, C.R. On discrete distributions arising out of methods of ascertainment. Calcutta pergamon press and statistical publishing society, 1965.

[2] Jain, K. Singh, H. and Bagai, I.Relations for reliability measures of weighted distributions. Comm. Statist. Theory Methods, 1989, 18, 4393-4412.

[3] Nanda, A. and Jatin, K. Some weighted distribution result on univariate bivariate cases. J.Statist. Plann. Inference, 1999, 77, 169-180.

[4] Misra , N., Gupta, N. and Dhariyal, I.D. Preservation of Some Aging Properties and Stochastic orders by Weighted Distributions. Communications in Statistics. Theory and Methods, 2008, 37, 627-644.

[5] Barlow, R.E. and Proschan, F. Statistical theory of reliability and life testing. New York: Holt, Rinhart and Winston, 1975.

[6] Bartoszewicz, J. and Skolimowska, M. Preservation of classes of life distributions and stochastic orders under weighting. Statist. Probab. Lett., 2006, 76, 587-596.

[7] Shaked, M. and Shanthikumar, J.G.Stochastic Orders. Springer, 2007.

[8] Ahmad and Kayid, Characterizations of the RHR and MIT orderings and the DRHR and IMIT classes of life distributions. Probability in the engineering and informational sciences, 2005, 19, Issue: 4. 